\title{
Local Hölder Regularity.of Weak Solutions for Singular Parabolic Systems of p-Laplacian Type
}

\author{
Khoirunisa Khoirunisa1, Corina Karim², M. Muslikh³ \\ 1,2,3 Department of Mathematics, Universitas Brawijaya
}

Email: khoir.n97@gmail.com,co_mathub@ub.ac.id,mslk@ub.ac.id

\begin{abstract}
Local Hölder regularity of weak solutions for degenerate parabolic systems of $p$-Laplacian type was proved by Corina, K. In this paper, we aim to show the local Hölder regularity of weak solutions in the singular case with $\frac{2 m}{m+2}<p<2, m \geq 2$, so that the Local Hölder regularity of weak solutions for parabolic systems of $p$-Laplacian type can hold for both cases. By applying Poincaré inequality, we show that its weak solutions within Hölder space, or we can tell that the local Hölder regularity for the singular case is valid.
\end{abstract}

Keywords: Hölder regularity; singular case; weak solutions

\section{INTRODUCTION}

Hölder regularity of weak solutions for parabolic equations in singular and degenerate case was introduced in [1] dan [2], where the coefficients are measurable and satisfy elliptic condition. However, the result in [2] is not showing the boundary estimates. After that, Bögelein was interested to prove for the boundary regularity from the previous result in [2], see [3]. Then, [4] investigated the same problem for nonlinear parabolic equations in the degenerate case. In 2002, DiBenedetto et al discussed about the regularity of weak solutions for quasilinear parabolic equations in singular and degenerate case to proving their Hölder character in [5]. The result in [2] was extended by Misawa to a larger class of right-hand side terms, but the singular case was ecluded here, see [6]. Meanwhile, for singular case, we can see the Hölder regularity in [7].

In addition, the Holder regularity of gradient solutions for all cases was proved in [8]. The study about Hölder regularity of gradients solution was continued in [9] for evolutionary p-Laplacian systems where the coefficients is Hölder continuous. Based on [10], the global weak solutions for similar case is exist. They used variational method to prove the existence of weak solutions globally. In 2018, Karim started to investigated about simpler p-Laplacian type in singular parabolic systems and showed that the weak solutions is bounded [11]. To prove the local boundedness in [11], we can adopt the method to prove energy estimates of singular parabolic equations in [12]. On the other hand, the existence of weak solutions in singular case was proved in [13] by using the Galerkin method. Furthermore, the intrinsic scaling method was used to treat the weak solutions to prove the Hölder regularity for degenerate case [14]. The intrinsic scaling 
method is based on [15], which used the intrinsic scaling method to approach the regularity in degenerate and singular partial differential equations. Motivated by the last result in[14], we would investigated the Hölder regularity of weak solutions in singular case.

Let $\Omega \subset \mathbb{R}^{n}$ be a bounded domain, $n \geq 2$, and $\partial \Omega$ is smooth boundary. The unknown function $u:(0, T) \times \Omega \rightarrow \mathbb{R}^{m}$ is vector valued function, $u=\left(u_{1}(z), u_{2}(z), \ldots, u_{m}(z)\right)$, where $z=\left(t, x_{1}, x_{2}, \ldots, x_{n}\right)$. Let $\frac{2 m}{m+2}<p<2, m \geq 2$, consider the parabolic systems

$$
\left\{\begin{array}{cc}
\partial_{t} u-\operatorname{div}\left(|D u|^{p-2} D u\right)=0 & \operatorname{in}(0, T) \times \Omega, \\
u(0, x)=u_{0}(x) & \text { on } \partial_{p}(0, T) \times \Omega,
\end{array}\right.
$$

where $u_{0}(x) \in W^{1, p}\left(\Omega, \mathbb{R}^{m}\right)$.

The result in [13] shows that for any initial condition, there exists weak solutions of (1) from $\Omega$ into $\mathbb{R}^{m}$. On the other hand, the local boundedness of the weak solution of (1) was proved by Karim in [11]. They modified the intrinsic scaling from the original work by DiBenedetto [12]. They used the intrinsic scaling for singular case. Their main theorem established that intrinsic scaling well-worked to prove the local boundedness of weak solution of (1).

We now turn to notion of Hölder continuous functions. For any $x, y \in \bar{\Omega}$, if $u \in$ $C^{0}(\bar{\Omega})$ satisfy

$$
\sup _{x, y \in \bar{\Omega}, x \neq y} \frac{|u(x)-u(y)|}{|x-y|^{\alpha}}<\infty,
$$

then $u \in C^{0, \alpha}(\bar{\Omega})$. Karim in [14] shows that the weak solutions of (1) in degenerate case satisfy the following theorem.

Theorem 1.[14] Let $p \geq 2$ and $u$ is weak solutions of (1) in $Q\left(\lambda^{2-p} \rho^{2}, \rho\right)\left(z_{0}\right)$. Then, $u$ is locally Hölder continuous with some $0<\alpha<1$. Furthermore, for any $Q_{3 \rho_{0}}\left(z_{0}^{\prime}\right) \subset Q$ with $z_{0}^{\prime} \in Q$ and $0<\rho_{0}<1$, there exist $C>0$ such that

$$
\left|u(z)-u\left(z^{\prime}\right)\right| \leq C\left\{\left|t-t^{\prime}\right|^{\frac{\alpha}{p}}+\left|x-x^{\prime}\right|^{\alpha}\right\},
$$

holds for any $z, z^{\prime} \in Q_{\rho_{0}}$ where $Q_{r}\left(z_{0}\right) \subset Q\left(\lambda^{2-p} \rho^{2}, \rho\right)\left(z_{0}\right) \subset Q_{\rho_{0}}\left(z_{0}^{\prime}\right) \subset Q_{3 \rho_{0}}\left(z_{0}^{\prime}\right)$.

Theorem 1 implies that the local Hölder regularity of weak solutions (1) in degenerate case is proved. Here, we aim to prove for the singular case, so that the local Hölder regularity of weak solutions (1) can be proven for both cases or $\frac{2 m}{m+2}<p<\infty$.

\section{METHODS}

We can prove the local Hölder regularity of weak solutions for parabolic systems of $p$ Laplacian type in singular case by showing that the weak solutions are elements of Hölder space. Our method is using Poincaré inequality to show that the weak solutions within Campanato space, then by isomorphism between Campanato and Hölder space, it is easy to see that its weak solutions are elements of Hölder space. The Poincaré inequality that we use is in the following theorem.

Theorem 2. (Poincaré Inequality).[16] Let $\Omega \subset \mathbb{R}^{n}$ be a bounded open set and $1 \leq p \leq$ $\infty$. Then, there exists positive constant $C=C(\Omega, p)$ so that

$$
|| u-\left.u_{\Omega}\right|_{L^{p}} \leq C|| D u||_{L^{p}}
$$


where $u_{\Omega}=\frac{1}{|\Omega|} \int_{\Omega} u d x$

\section{RESULTS AND DISCUSSION}

For singular equation (1) with $1<p<2$, we have the cylinder of the type

$$
Q(\rho)\left(z_{0}\right)\left(t_{0}-\rho^{2}, t_{0}+\rho^{2}\right) \times B\left(\lambda^{\frac{p-2}{2}} \rho, x_{0}\right) .
$$

By switching the cylinder $Q(\rho)\left(z_{0}\right)$ to $Q(1)$, we have

$$
v(s, y)=\frac{u\left(t_{0}+\lambda^{2-p} \rho s, x_{0}+\rho y\right)}{\lambda \rho} ;(s, y) \in B(1) \times(-1,1) \equiv Q(1) .
$$

Suppose that on such a certain cylinder the relations

$$
\frac{1}{\left|Q(\rho)\left(z_{0}\right)\right|} \int_{Q(\rho)\left(z_{0}\right)}|D u|^{p} d z \approx \lambda^{p}
$$

Hence, we have

in $Q(\rho)\left(z_{0}\right)$.

$$
\partial_{t} u-\lambda^{p-2} \operatorname{div}(D u)=0
$$

Let $u \in L^{\infty}\left(0, T ; W^{1, p}\left(\Omega, \mathbb{R}^{m}\right)\right)$ be a weak solutions of $(1)$ in cylinder $Q=(0, T) \times \Omega$ where $z_{0}=\left(t_{0}, x_{0}\right) \in Q, \lambda \geq 1$ and $0<\rho<1$ such that $Q\left(\rho^{2}, \lambda^{\frac{p-2}{2}} \rho\right) \subset Q$. While, our main theorem is the following.

Theorem 3.Let $\frac{2 m}{m+2}<p<2, m \geq 2$ and $\mathrm{u}$ is a weak solution of $(1)$ in $Q\left(\rho^{2}, \lambda^{\frac{p-2}{2}} \rho\right)\left(z_{0}\right)$. Then $u$ is locally $\alpha$-Hölder continuous with some $0<\alpha<1$. Furthermore, for any $Q_{3 \rho_{0}}\left(z_{0}^{\prime}\right) \subset Q$ with $z_{0}^{\prime} \in Q$ and $0<\rho_{0}<1$, there $C>0$ such that

$$
\left|u(z)-u\left(z^{\prime}\right)\right| \leq C\left\{\left|t-t^{\prime}\right|^{\frac{\alpha}{p}}+\left|x-x^{\prime}\right|^{\alpha}\right\},
$$

holds for any $z, z^{\prime} \in Q_{\rho_{0}}\left(z_{0}^{\prime}\right)$ where $Q_{r}\left(z_{0}\right) \subset Q\left(\rho^{2}, \lambda^{\frac{p-2}{2}} \rho\right)\left(z_{0}\right) \subset Q_{\rho_{0}}\left(z_{0}^{\prime}\right) \subset Q_{3 \rho_{0}}\left(z_{0}^{\prime}\right)$

\section{Proof.}

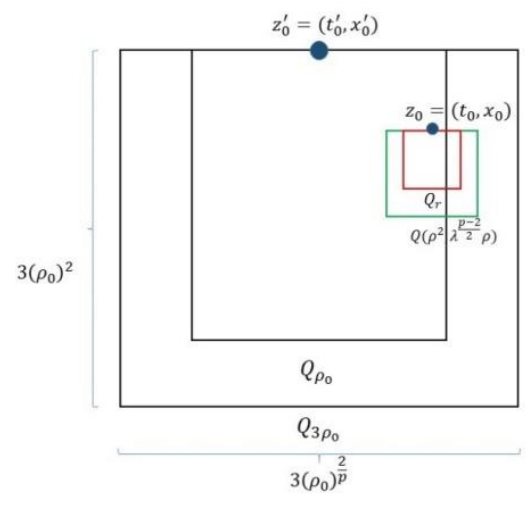

Figure 1. Any cylinder in $Q$ 
Let $Q_{3 \rho_{0}}\left(z_{0}^{\prime}\right):=Q\left(3\left(\rho_{0}\right)^{2}, 3\left(\rho_{0}\right)^{\frac{2}{p}}\right) \subset Q$ is any cylinder centered in $z_{0}^{\prime}=\left(t_{0}^{\prime}, x_{0}^{\prime}\right) \in Q$, and $0<\rho_{0}<1$. Let $z_{0}=\left(t_{0}, x_{0}\right) \in Q_{\rho_{0}}\left(z_{0}^{\prime}\right):=Q\left(\left(\rho_{0}\right)^{2},\left(\rho_{0}\right)^{\frac{2}{p}}\right)\left(z_{0}^{\prime}\right)$, where $\lambda \geq 1$. It is easy to see that

$$
\int_{Q_{r}\left(z_{0}\right)}|D u|^{p} d z \leq\left|Q_{r}\left(z_{0}\right)\right| \frac{1}{\left|Q\left(\rho^{2}, \lambda^{\frac{p-2}{2}} \rho\right)\left(z_{0}\right)\right|} \int_{Q\left(\rho^{2}, \lambda^{\frac{p-2}{2}} \rho\right)\left(z_{0}\right)}|D u|^{p} d z
$$

By using relation (3) we have

$$
\frac{1}{\left|Q_{r}\left(z_{0}\right)\right|} \int_{\left.Q_{r}\left(z_{0}\right)\right)}|D u|^{p} d z \leq C r^{m+p \alpha}
$$

where $0<r<1,0<\alpha<1$, and $C=\pi r^{2-m}$.

Next, recall the Poincaré inequality. Then, substitute (4) to (2) we have

$$
\begin{aligned}
\int_{Q_{r}\left(z_{0}\right)}\left|u-u_{r}\right|^{p} d z & \leq C r^{p} \int_{Q_{r}\left(z_{0}\right)}|D u|^{p} d z \\
& \leq C r^{m+p+p \alpha}
\end{aligned}
$$

holds for any $z_{0} \in Q_{\rho_{0}}\left(z_{0}\right)$ and $0<r<2\left(\rho_{0}\right)^{\frac{2}{p}}$.

We apply the characterization between Hölder continuous functions and Campanato space, which implies that for $u \in L^{p}\left(Q_{\rho_{0}}\left(z_{0}^{\prime}\right)\right)$ and

then

$$
\sup _{Q_{\rho_{0}}\left(z_{0}^{\prime}\right)} \frac{1}{r^{m+p+p \alpha}} \int_{Q_{r}\left(z_{0}\right)}\left|u-u_{r}\right|^{p} d z \leq C,
$$

$$
u \in C^{0, \alpha}\left(Q_{\rho_{0}}\left(z_{0}^{\prime}\right)\right)
$$

Thus, we have for any two points $\left(t_{1}, x_{1}\right),\left(t_{2}, x_{2}\right) \in Q_{\rho_{0}}\left(z_{0}^{\prime}\right)$ with $\left|t_{1}-t_{2}\right|=r^{p}$,

$$
\left|u\left(t_{1}, x_{1}\right)-u\left(t_{2}, x_{1}\right)\right| \leq C\left|t_{1}-t_{2}\right|^{\frac{\alpha}{p}}
$$

and let $\left|x_{1}-x_{2}\right|=r$, then

$$
\left|u\left(t_{2}, x_{2}\right)-u\left(t_{2}, x_{1}\right)\right| \leq C\left|x_{1}-x_{2}\right|^{\alpha} .
$$

Moreover, we can conclude from (6),(7) and triangle inequality that

$$
\left|u(z)-u\left(z^{\prime}\right)\right| \leq C\left\{\left|t-t^{\prime}\right|^{\frac{\alpha}{p}}+\left|x-x^{\prime}\right|^{\alpha}\right\},
$$

in $Q_{\rho_{0}}\left(z_{0}^{\prime}\right)$ or we have 


$$
u \in C^{\prime} \frac{\alpha}{p^{\prime}} \alpha\left(Q_{\rho_{0}}\left(z_{0}^{\prime}\right)\right)
$$

Since, $Q_{3 \rho_{0}}\left(z_{0}^{\prime}\right) \subset Q$ is arbitrary, then $u \in C_{l o c}^{\frac{\alpha}{p^{\alpha}} \alpha}\left(Q, \mathbb{R}^{n}\right)$. By this result, we can tell that the local Hölder regularity of weak solutions for parabolic systems of $p$-Laplacian type in singular case is proved.

\section{CONCLUSIONS}

Based on the previous results and discussion, it can be concluded that in singular case the weak solutions for parabolic systems of $p$-Laplacian type are elements of Hölder space, $u \in C^{\prime, \frac{\alpha}{p^{\prime}}, \alpha}\left(Q_{\rho_{0}}\left(z_{0}^{\prime}\right)\right)$. Since $Q_{3 \rho_{0}}\left(z_{0}^{\prime}\right) \subset Q$ is arbitrary, then $u \in C_{\text {loc }}^{\frac{\alpha}{p^{\alpha} \alpha}}\left(Q, \mathbb{R}^{n}\right)$ or we can say that the local Hölder regularity of weak solutions is proved.

\section{ACKNOWLEDGEMENT}

This paper partially supported by the doctoral grant No. 44/UN10.F09/PN/2020 at Mathematics and Natural Sciences Faculty, Universitas Brawijaya.

\section{REFERENCES}

[1] C. Ya-Zhe and E. DiBenedetto, "Hölder estimates of solutions of singular parabolic equations with measurable coefficients," Archive for rational mechanics and analysis, vol. 118, no. 3, pp. 257-271, 1992.

[2] E. DiBenedetto, "On the local behaviour of solutions of degenerate parabolic equations with measurable coefficients," Annali della Scuola Normale Superiore di Pisa-Classe di Scienze, vol. 13, no. 3, pp. 487-535, 1986.

[3] V. Bögelein, "Global gradient bounds for the parabolic p-Laplacian system," Proceedings of the London Mathematical Society, vol. 111, no. 3, pp. 633-680, 2015.

[4] M. M. Porzio and V. Vespri, "Holder estimates for local solutions of some doubly nonlinear degenerate parabolic equations," Journal of differential equations, vol. 103, no. 1, pp. 146-178, 1993.

[5] E. DiBenedetto, J. M. Urbano, and V. Vespri, "Current issues on singular and degenerate evolution equations," in Handbook of Differential Equations: Evolutionary Equations, vol. 1, Elsevier, 2002, pp. 169-286.

[6] M. Misawa, "A Hölder estimate for nonlinear parabolic systems of p-Laplacian type," Journal of Differential Equations, vol. 254, no. 2, pp. 847-878, 2013.

[7] C. Karim and M. Misawa, "Hölder regularity for singular parabolic systems of \$ p \$Laplacian type," Advances in Differential equations, vol. 20, no. 7/8, pp. 741-772, 2015.

[8] C. Karim and M. Misawa, "Gradient Hölder regularity for nonlinear parabolic systems of \$ p \$-Laplacian type," Differential and Integral equations, vol. 29, no. 3/4, pp. 201-228, 2016.

[9] M. Misawa, "Local Hölder regularity of gradients for evolutional p-Laplacian systems," Annali di Matematica Pura ed Applicata, vol. 181, no. 4, pp. 389-405, 2002. 
[10] C. Karim and M. Misawa, "Existence of global weak solutions for Cauchy-Dirichlet problem for evolutional p-Laplacian systems," in AIP Conference Proceedings, 2017, vol. 1913, no. 1, p. 020001.

[11] C. Karim, "Local Boundedness of weak solutions for singular parabolic systems of p-Laplacian type," The Australian Journal of Mathematical Analysis and Applications, vol. 15, no. 2-8, pp. 1-5, 2018.

[12] E. DiBenedetto, Degenerate parabolic equations. Springer Science \& Business Media, 2012.

[13] P. R. Akbar, C. Karim, and R. B. EW, "The Existence of Global Weak Solutions for Singular Parabolic System of p-Laplacian Type," in Journal of Physics: Conference Series, 2020, vol. 1562, no. 1, p. 012009.

[14] C. Karim, "Local Hölder regularity of weak solutions for degenerate parabolic type," in AIP Conference Proceedings, 2019, vol. 2192, no. 1, p. 050003.

[15] J. M. Urbano, "The method of intrinsic scaling," Lecture Notes in Mathematics, vol. 1930, 1930.

[16] B. Dacorogna, Introduction to the Calculus of Variations. World Scientific Publishing Company, 2014. 